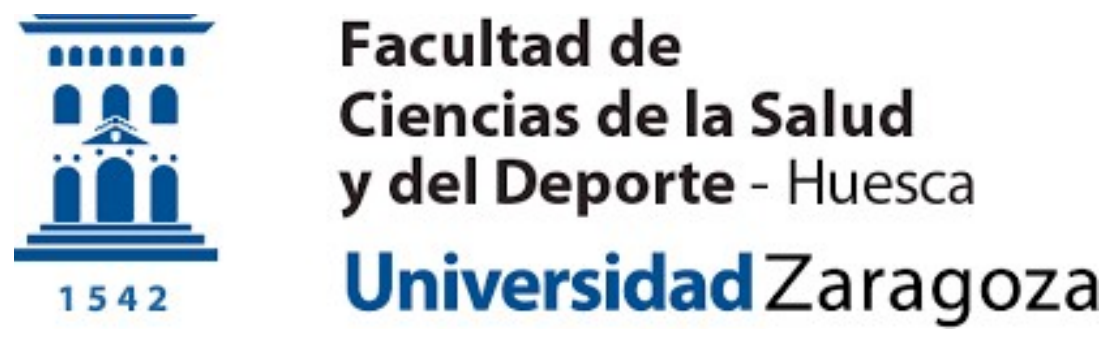

Master Universitario en Evaluación y Entrenamiento

Físico para la Salud

\title{
Análisis de marcadores de salud en el trail running
}

\section{Analysis of health markers in trail running}

Daniel Fernandez de Landa Nicolau

Director/es

Carlos Castellar Otín

Francisco Pradas de Fuente

Carlos Peñarrubia Lozano

Facultad de Ciencias de la Salud y del Deporte 


\section{Resumen}

Introducción. Los ultramaratones de montaña son competiciones cada vez más populares y extendidas entre la población. La participación en estas pruebas puede tener un gran efecto agudo que puede conllevar efectos secundarios nocivos sobre el organismo.

Objetivos. El objetivo es analizar el impacto agudo producido en el organismo durante una carrera de montaña en corredores aficionados analizando marcadores sanguíneos y valorando la posible pérdida de fuerza en las extremidades inferiores tras la prueba.

Método. Se trata de un estudio cuasiexperimental pre-post de nueve corredores adultos $(47,8 \pm 4,4$ años), con un IMC de $23,4 \pm 2,5$ y un porcentaje de masa grasa de $11,1 \pm 2,2$. Se realizó en la Ultra-Trail Guara Somontano, con 108 km y 5800 metros de desnivel positivo acumulado. Para analizar la respuesta fisiológica se analizaron diferentes marcadores sanguíneos ( $\mathrm{Na}, \mathrm{CPK}$, bilirrubina, ALT, creatinina y leucocitos), y para conocer el impacto físico se evaluó la fuerza de las extremidades inferiores utilizando test de saltos (SJ, CMJ y ABK). Para calcular la significación estadística entre valores pre y post carrera se utilizó el test T para muestras relacionadas y para valorar la posible influencia de algunos factores sobre las variables medidas se utilizó el test ANOVA.

Resultados. Se observaron incrementos significativos en la CPK $(155,89 \pm 65,28 \mathrm{UI} / \mathrm{L}$ vs $2492 \pm 844,64 \mathrm{UI} / \mathrm{L}(p=, 008))$, bilirrubina $(0,66 \pm 0,24 \mathrm{mg} / \mathrm{dL}$ vs $1,14 \pm 0,28 \mathrm{mg} / \mathrm{dL}(p=$ ,030)) ALT $(20,33 \pm 4,97 \mathrm{UI} / \mathrm{L}$ vs $29,11 \pm 6,47 \mathrm{UI} / \mathrm{L}(p=, 012))$, creatinina $(0,81 \pm 0,15 \mathrm{mg} / \mathrm{dL}$ vs $1,29 \pm 0,38 \mathrm{mg} / \mathrm{dL}(p=, 008)$ y leucocitos $(5,31 \pm 1,43 \times 109 / \mathrm{L}$ vs $13,07 \pm 3,25 \times 109 / \mathrm{L}(p$ $=, 008))$, y decrementos significativos en SJ $(23,8 \pm 2,46$ vs $15,99 \pm 3,61(p=, 008))$, CMJ $(27,84 \pm 3,12$ vs $18,84 \pm 4,34(p=, 008))$ y $\operatorname{ABK}(32,03 \pm 5,50$ vs $24,12 \pm 4,82(p=, 008))$.

Conclusiones. Los resultados obtenidos parecen indicar que este tipo de pruebas generan un elevado impacto muscular junto a una disfunción renal y hepática aguda. Una mayor cantidad de horas de entrenamiento, podría ser un buen factor para minimizar dicho impacto.

Palabras clave: ultra resistencia, fatiga, marcadores sanguíneos, saltos. 


\section{Abstract}

Introduction. Mountain ultramarathons are increasingly popular and widespread competitions among the population. Participation in this kind of events can have an acute effect that can lead to harmful side effects on the body.

Objectives. The aim is to analyze the acute impact produced on the body during a mountain race in amateur runners by analyzing blood markers and evaluating the possible loss of lower limb strength after the race.

Method. This is a quasi-experimental pre-post study of nine adult runners (47.8 \pm 4.4 years), who had a BMI of $23.4 \pm 2.5$ and a percentage of fat mass of $11.1 \pm 2,2$. The race where the study was carried out was the Ultra-Trail Guara Somontano (UTGS) with $108 \mathrm{~km}$ and 5800 meters of accumulated positive slope. To analyze the physiological response, different blood markers ( $\mathrm{Na}, \mathrm{CPK}$, bilirubin, ALT, creatinine and leukocytes) were analyzed, while the strength of the lower limbs was evaluated for the physical impact by using some jump tests (SJ, CMJ and ABK). T test for related samples was used to calculate the statistical significance between pre- and post-race values, and the ANOVA test was used to assess the possible influence of some factors on the measured variables.

Results. Significant increases in CPK were observed $(155.89 \pm 65.28 \mathrm{IU} / \mathrm{L}$ vs $2492 \pm$ $844.64 \mathrm{IU} / \mathrm{L}(\mathrm{p}=.008))$, bilirubin $(0.66 \pm 0.24 \mathrm{mg} / \mathrm{dL}$ vs $1,14 \pm 0.28 \mathrm{mg} / \mathrm{dL}(p=.030))$ $\operatorname{ALT}(20.33 \pm 4.97 \mathrm{IU} / \mathrm{L}$ vs $29.11 \pm 6.47 \mathrm{IU} / \mathrm{L}(p=.012))$, creatinine $(0,81 \pm 0.15 \mathrm{mg} /$ $\mathrm{dL}$ vs $1.29 \pm 0.38 \mathrm{mg} / \mathrm{dL}(\mathrm{p}=.008))$ and leukocytes $(5.31 \pm 1.43 \times 109 / \mathrm{L}$ vs $13.07 \pm$ $3.25 \times 109 / L(p=.008))$, and significant decreases in SJ (23.8 \pm 2.46 vs. $15.99 \pm 3.61$ $(p=.008))$, CMJ $(27.84 \pm 3.12$ vs. $18.84 \pm 4.34(p=.008))$ and $A B K(32.03 \pm 5.50$ vs. $24.12 \pm 4.82(p=.008))$.

Conclusions. The obtained results seem to indicate that this kind of events generate a high muscular impact with acute kidney and liver dysfunction. A greater number of hours of training, could be a good factor to minimize that impact

Key words. Ultra-endurance, fatigue, blood markers and jumps. 


\section{Tabla de contenido}

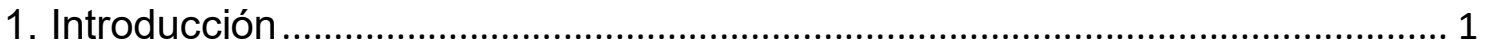

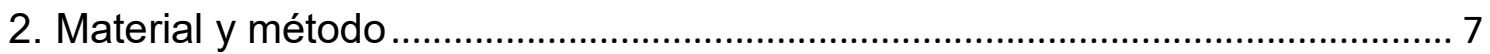

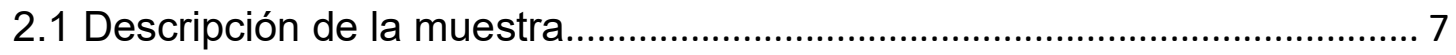

2.2 Descripción de la prueba........................................................................... 7

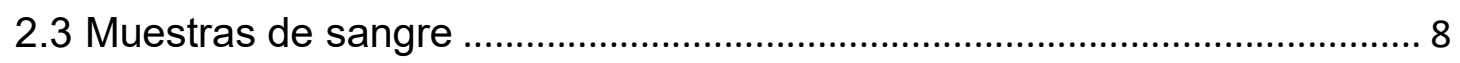

2.4 Evaluación de la fuerza y composición corporal ........................................... 9

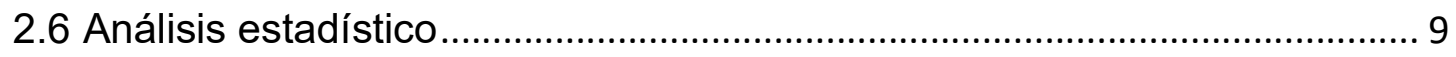

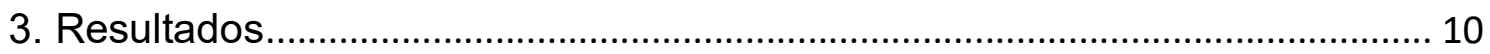

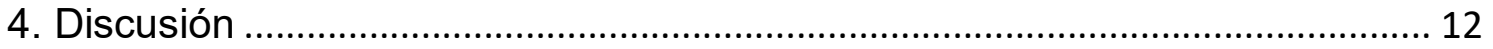

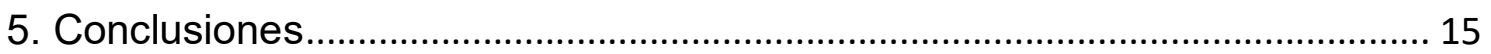

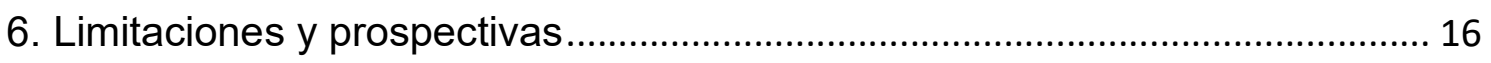

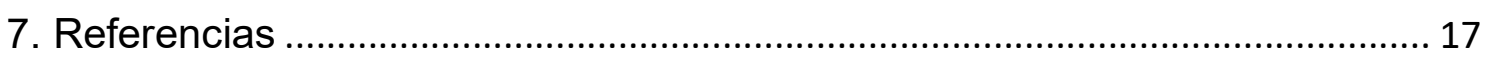




\section{Introducción}

Los eventos de ultra resistencia, concretamente los eventos de ultramaratón, han ganado mucha popularidad y participación en los últimos 25 años (1). La tendencia de participación mundial total y el rendimiento de los corredores, han aumentado exponencialmente, especialmente entre las mujeres (2).

La referencia más antigua encontrada hasta la fecha de carreras por montaña es la de las fell races de Escocia, entre los años 1040 y 1064 (3). En Estados Unidos, el Trail running nació en 1904 cuando unos corredores de San Francisco recorrieron la distancia existente entre Dipsea Inn y Mount Tamalpais (San Francisco, California), denominándola Dipsea race (3).

En España, las primeras referencias a este tipo de carreras provienen del País Vasco a principios del siglo XX, donde los participantes ascendían el monte atados de las manos. En la Sierra de Guadarrama encontramos la primera referencia de carreras de Trail running en el año 1916, cuando una sociedad organizó la primera prueba de esta modalidad de la que ha quedado constancia. El recorrido era Cercedilla-siete picosCercedilla. Desde entonces, hasta 1935 surgieron más pruebas en la Sierra de Guadarrama, pero con el inicio de la guerra civil desaparecieron hasta 1986 (3).

En la actualidad existen multitud de carreras de trail running en todo el mundo siendo la más mediática la Ultra-Trail de Mont Blanc o UTMB (Chamonix-Mont-Blanc, Francia), cuya participación está reservada para los mejores corredores de la temporada.

Según la Asociación Internacional de Trail Running (ITRA), podríamos definir el trail running como una carrera pedestre en la que puede participar cualquier persona, en un entorno natural (montaña, desierto, bosque, llanura...), con un mínimo de carretera asfaltada o pavimentada (que no debe exceder el $20 \%$ del recorrido total), sin inclinaciones mayores del $40 \%$ o dificultades técnicas de II grado y en una sola etapa. El terreno puede variar (camino de tierra, sendero forestal, pista única...), y la ruta debe estar debidamente marcada. Esto significa que los corredores recibirán suficiente información para completar la carrera sin perderse. Dicha información incluye marcas físicas (banderas, cintas, letreros...), o marcas permanentes GR (Senderos de Gran Recorrido), pistas de GPS (Global Position System), o indicaciones de mapa. Se prestará especial atención en el marcaje de cruces y otros lugares de posibles pérdidas y siempre que sea necesario la organización ubicará físicamente alguna persona en esos lugares. Existirá como mínimo un indicador kilométrico cada 10 kilómetros, con la cifra kilométrica bien definida (4). 
Según la Real Federación Española de Atletismo, las pruebas con una distancia de hasta $42 \mathrm{~km}$ se denominan trail y las pruebas que superen los $42 \mathrm{~km}$ de distancia se denominan ultra trail, y se subdividen según la distancia. Carreras de entre 42 y $69 \mathrm{~km}$ son ultra trail medio, de entre 70 y $99 \mathrm{~km}$ son ultra trail largo y carreras iguales o superiores a $100 \mathrm{~km}$ son ultra trail XL.

A pesar de que la popularidad de este deporte está creciendo exponencialmente, no existen muchos artículos que hayan analizado marcadores de salud en el trail running (5). La mayoría de las publicaciones se centran en pruebas en terrenos convencionales y el impacto que estas pruebas tienen sobre el organismo (6), no es comparable con el impacto que generan las pruebas en montaña, en las cuales el terreno irregular y la acumulación de desnivel las hace más duras (7).

Algunas de las complicaciones asociadas a las carreras de ultra distancia que se han ido analizando en los últimos años son la deshidratación, el daño muscular, disfunciones hepáticas y renales y alteraciones en el sistema inmune. La mayor parte de los autores focalizan sus estudios en analizar el daño muscular y la inflamación que se produce. La fosfocreatinkinasa (CPK) y la enzima lactato deshidrogenasa (LDH), ambas analizadas en plasma, y algunas interleukinas, son los marcadores más utilizados para medir la inflamación, y por lo tanto el daño muscular (8). En el caso de la deshidratación, todos los autores revisados coinciden en analizar la concentracion de sodio en plasma para valorar el daño producido por la deshidratación (9). Para analizar las disfunciones hepáticas la mayoria de autores utilizan como marcadores de daño algunas enzimas hepáticas, como la alanina aminotrasferasa (ALT), aspartato aminotrasferasa (AST) y fosfatasa alcalina (ALP) y la bilirrubina, tanto la total como la directa (10). En el caso de la disfuncion renal el marcador mas utilizado por los autores es la creatinina plasmática, seguido por el nitrógeno ureico en plasma (BUN) (11). Por último, para analizar una alteración en el sistema inmune, los marcadores que más se utilizan son los leucocitos y las citoquinas quimiotácticas. Algunas de las más habitualmente analizadas son la interleukina 8, la proteína 10 inducida por interferón gamma (IP-10), las células T normales y la eotaxina, que están involucradas en el reclutamiento de leucocitos (12).

Teniendo en consideración la información reflejada en la literatura científica, resulta de interés para la comunidad científica analizar y valorar una serie de parámetros sanguíneos representativos de la salud de los corredores. Uno de ellos son los niveles de hiponatremia durante la carrera y la deshidratación midiendo las concentraciones de sodio en plasma. Por otro lado, para la valoración del daño muscular producido por las contracciones repetitivas que se producen al correr, se ha tenido en consideración el 
comportamiento de la CPK, mientras que para valorar la destrucción de hematíes se ha medido la bilirrubina en plasma. En referencia a la evaluación de la respuesta del sistema inmunitario de los corredores, se suelen analizar los leucocitos en plasma. Por último, para valorar el correcto funcionamiento del hígado y del riñón se han analizado diferentes enzimas, siendo las más habituales la alanina aminotransferasa y la creatinina en plasma respectivamente.

\section{Hiponatremia}

Con respecto a las concentraciones de sodio en plasma, sus valores han sido medidos para controlar el estado de hiponatremia en el que llegan los corredores a meta, o si la sufren durante la carrera, ya que la hiponatremia asociada al ejercicio (EAH), suele estar presente en pruebas de larga distancia (9). La EAH se define como una concentración de sodio menor a $135 \mathrm{mmol} / \mathrm{L}$ en plasma durante la actividad o después de 24 horas de realizarla. La EAH normalmente sucede debido a una ingesta excesiva de líquido hipotónico (agua), junto con una alta tasa de excreción de sodio mediante el sudor, pero se han identificado otros factores de riesgo como la composición del líquido ingerido, el tiempo de carrera, el IMC y la temperatura media de la prueba $(9,13)$. Esta situación provoca un desplazamiento del líquido extracelular al interior de las células para igualar las concentraciones de sodio, provocando una posible inflamación celular que se manifiesta con cuadros de náuseas, vómitos, confusión y congestión pulmonar (14).

En una situación de reposo, el sistema renal sería capaz de revertir esta situación, excretando agua y reabsorbiendo sodio para elevar la concentración del mismo; pero durante el ejercicio predominan los mecanismos simpáticos, que junto a la activación del sistema renina-angiotensina-aldosterona reducen el flujo de orina para impedir un descenso de la tensión arterial. De esta manera, el riñón no es capaz de excretar liquido hipotónico para corregir la concentración de sodio en plasma, produciéndose hiponatremia (14).

\section{Daño muscular}

La CPK, es una enzima presente en las células musculares que, ante la ruptura de las mismas debido al estrés y daño muscular producido por los constantes y repetitivos impactos al correr, se libera al torrente sanguíneo junto con más proteínas pudiendo producir necrosis celular. Si la concentración de CPK en plasma supera 1000-5000 UI/L significa que se ha producido rabdomiólisis producida por el ejercicio $(8,15-18)$. La CPK se encarga de refosforilar ATP a partir de las reservas de fosfocreatina en el músculo, por lo que es muy importante para un rendimiento óptimo. El rango estándar de referencia en no deportistas es en mujeres de 0,5 a 1,0 mg/dl y en hombres de 0,7 a 1,2 
$\mathrm{mg} / \mathrm{dl}$. En atletas se han hallado valores de referencia y se ha determinado que los atletas masculinos tienen la CPK en suero más elevada que las atletas femeninas (16). A mayor duración e intensidad del ejercicio se producen mayores niveles de CPK en sangre y por lo tanto mayor daño muscular $(8,17,19)$, lo que se traduce en mayores niveles de mioglobina en sangre, aumentando la probabilidad de insuficiencia renal debida a rabdomiólisis por ejercicio.

\section{Hemólisis y bilirrubina}

La bilirrubina es un producto de la degradación de los hematíes, por lo que valores elevados en sangre de bilirrubina después de la prueba puede significar altos niveles de destrucción de hematíes. En el caso de corredores de ultra distancia puede ser debido a los continuos microtraumatismos producidos en los pies a la hora de correr, al recambio fisiológico, al catabolismo de la hemoglobina o por una lesión hepática (17). La hemólisis en la sangre es mayor cuanto más intensa o larga es la prueba.

\section{Marcadores de daño hepático}

Los datos que evalúan la lesión hepática usando enzimas como la ALT durante una ultramaratón son controvertidos. La ALT aumenta significativamente después de una ultramaratón, pero la tasa de aumento no fsuele ser superior al de otras enzimas analizadas, lo que sugiere que la masa muscular se encuentra más dañada que el hígado (8). Sin embargo, los aumentos en enzimas hepáticas como ALT, AST y g-GTP en ultramaratones de larga distancia indica lesión hepática y muscular, siendo el grado de lesión hepática proporcional a la carga de trabajo (10).

Esta lesión hepática, debida a la ultra distancia, podría estar relacionada con cambios en la membrana de las células hepáticas por la peroxidación lipídica debido al flujo sanguíneo deteriorado y la liberación de radicales libres. Lo que indica una disminución temporal de la función hepática durante el tiempo que dura la prueba (17).

\section{Creatinina}

La creatinina es un compuesto orgánico producido a partir de la creatina y que en condiciones normales es filtrada en el riñón para ser excretada a través de la orina. Cuando se realiza una ultramaratón de montaña, un factor bastante común es la deshidratación. En un estado de hipovolemia por deshidratación disminuye la perfusión sanguínea en la nefrona, lo que disminuye la tasa de filtración glomerular (TFG), y puede llegar a producir insuficiencia renal aguda (IRA). Esto provoca que la creatinina no pueda ser excretada en la orina, acumulándose en el plasma y siendo un buen indicador de un mal funcionamiento del riñón (20). Además, también puede aparecer insuficiencia renal 
debida a la liberación al torrente sanguíneo de todas las sustancias producidas en la destrucción de los miocitos por isquemia, y por los continuos traumatismos y daño muscular ocasionados al correr, fenómeno conocido como rabdomiólisis (18).

Un adecuado marcador para conocer el nivel de lesión renal es la concentración de nitrógeno ureico En carreras de $100 \mathrm{~km}$ de distancia, donde la intensidad es media alta, la concentración de nitrógeno ureico es más alta en comparación con carreras de distancias más cortas o más largas (en maratón la intensidad es más alta pero la duración es menor y en carreras de más distancia la intensidad es más baja, por lo que no se produce tanto daño a nivel celular) (17),

\section{Leucocitos y sistema inmune}

Aunque el aumento en el número de leucocitos a menudo es indicativo de infección y/o inflamación, se sabe que la leucocitosis inducida por el ejercicio es un fenómeno transitorio, con recuentos normales en los subtipos de leucocitos y que generalmente regresan a los niveles previos al ejercicio dentro de las 6 a 24 horas posteriores al cese del ejercicio (21). Las células predominantes movilizadas en la práctica deportiva son los neutrófilos y los linfocitos. A los 30-60 minutos después de finalizar la actividad, se produce una rápida reducción de linfocitos en sangre, denominada como linfocitopenia, provocando una neutrofilia sostenida (recuento elevado de neutrófilos en sangre) (21).

Las causas de la leucocitosis inducida por el ejercicio son multifactoriales, pero entre las más importantes, destacan el aumento de la hemodinámica y la actuación de catecolaminas (21). El ejercicio agudo produce aumento del gasto cardíaco, de la vasodilatación vascular y del flujo sanguíneo, que ejercen mayores fuerzas mecánicas sobre el endotelio, provocando que los leucocitos se separen del endotelio y entren en circulación. Además, el aumento del flujo linfático con el ejercicio también puede contribuir a esta leucocitosis a medida que la linfa se vacía en la sangre a través del conducto torácico. Otro factor por el cual se produce el aumento de leucocitos es el aumento de la actividad del sistema nervioso simpático y la secreción resultante de catecolaminas, que se sabe que tienen un profundo efecto sobre el tráfico de leucocitos (21).

En este sentido, factores que aumentan los niveles de leucocitos en pruebas de ultra maratón son la inflamación y el daño muscular que se produce por las contracciones repetitivas al correr. Estudios como el efectuado por Shin et al. (12), demuestran que las concentraciones de neutrófilos aumentan durante y después del ejercicio, mientras que las concentraciones de linfocitos se incrementan durante el ejercicio, disminuyendo por debajo de los valores previos después de un esfuerzo físico de larga duración, 
Master Universitario en Evaluación y Entrenamiento Físico para la Salud.

corroborando la neutrofilia, la monocitosis y la linfocitopenia inducidas por el ejercicio que se han comentado anteriormente.

\section{Influencia de la fuerza en las carreras de montaña}

La fuerza es un factor muy importante en el rendimiento de los deportes de resistencia (22-24). Los entrenamientos de fuerza convencionales o incluso entrenamientos más novedosos, como entrenamientos de fuerza donde se combinan ejercicios explosivos y de pliometría, combinados con los entrenamientos de resistencia (entrenamiento concurrente), generan mayores mejoras en el rendimiento de los deportistas que el entrenamiento de resistencia únicamente (23-25). Esto es debido a que se produce una mejora en la economía de carrera (el consumo de oxígeno requerido a una velocidad de carrera submáxima dada), de los corredores $(22,25)$, es decir, el consumo de oxígeno que requieren para llevar una velocidad de carrera determinada es menor, siendo más eficiente y provocando que la aparición de la fatiga se retrase. En este sentido, el daño muscular que se produce durante las carreras de ultra montaña y la fatiga acumulada, pueden afectar a la economía de carrera (22), disminuyendo el rendimiento del corredor o incluso pudiendo provocar lesiones músculo tendinosas agudas $(26,27)$.

El hecho de que estas pruebas hayan experimentado un aumento tan grande en popularidad, supone que los investigadores estén tratando de dilucidar más evidencias para poder analizar en mayor profundidad el impacto sobre la salud que suponen estas pruebas (28), o, en el caso de corredores profesionales, tratar de mejorar el rendimiento o la recuperación (29).

Teniendo en cuenta el estrés físico al que se ve sometido al organismo durante la realización de una ultra trail (15), se puede pensar que su práctica de manera no planificada o controlada puede tener consecuencias negativas para la salud. Siguiendo esta línea, sería necesario conocer hasta qué punto la respuesta adaptativa fisiológica del organismo es resultado del ejercicio intenso, o está indicando que el corredor pueda requerir atención médica específica (18).

El objetivo de este estudio es analizar el impacto agudo producido en el organismo durante una carrera de montaña en corredores aficionados, analizando diferentes marcadores bioquímicos sanguíneos ( $\mathrm{Na}, \mathrm{CPK}$, bilirrubina, ALT, creatinina y leucocitos) y valorando la posible pérdida de fuerza producida sobre las extremidades inferiores. 


\section{Material y método}

\subsection{Descripción de la muestra}

Se trata de un estudio cuasiexperimental con un diseño pre-post de nueve corredores varones de $47,8 \pm 4,4$ años de edad, con una experiencia en este tipo de pruebas de resistencia y ultra-resistencia en montaña de 5,9 $\pm 2,9$ años. Los deportistas entrenaban $8,3 \pm 2$ horas semanales con un desnivel acumulado de $46595 \pm 23810$ metros positivos a lo largo de la temporada. Las características de la muestra se presentan en la tabla 1. La participación de los sujetos fue voluntaria, firmando el consentimiento informado avalado por el Comité Ético de Investigación Clínica de Aragón (Acta N 16/2017 del CEIC Aragón).

Tabla 1. Características de la muestra

\begin{tabular}{|c|c|}
\cline { 2 - 2 } \multicolumn{1}{c|}{} & Media \pm DS \\
\hline Edad (años) & $47,8 \pm 4,4$ \\
\hline Altura $(\mathrm{cm})$ & $178,6 \pm 7$ \\
\hline Peso $(\mathrm{kg})$ & $74,9 \pm 10,4$ \\
\hline $\mathrm{IMC}\left(\mathrm{kg} / \mathrm{m}^{2}\right)$ & $23,4 \pm 2,5$ \\
\hline$\% \mathrm{GCT}$ & $11,1 \pm 2,2$ \\
\hline Entrenamiento semanal (horas) & $8,3 \pm 2$ \\
\hline Desnivel acumulado anual (m/año) & $46595 \pm$ \\
23810 \\
\hline Experiencia en carreras de montaña \\
(años) & $5,9 \pm 2,9$ \\
\hline
\end{tabular}

IMC: índice de masa corporal; \%GCT: porcentaje de grasa corporal total; DS: desviación estándar

\subsection{Descripción de la prueba}

El estudio se llevó a cabo durante la realización de la Ultra-Trail Guara Somontano (UTGS). Esta competición se realiza por las sierras contiguas a la población de Alquezar (Huesca), y discurre por los caminos de montaña y sendas del Parque Natural de los Cañones y la Sierra de Guara. Tiene una distancia total de 108 km y 5800 metros de desnivel positivo acumulado. El tiempo máximo determinado por la organización para completar la prueba es de 26 horas. 


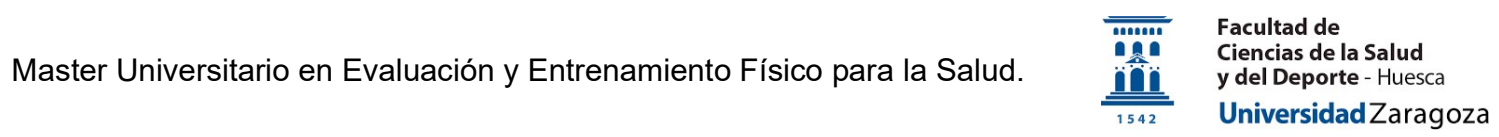

Todo el recorrido transcurre por senderos y pistas claramente visibles y marcadas debidamente por la organización, atravesando numerosas localidades y presentando diez puntos de avituallamiento tanto líquido como sólido.

La temperatura media durante la prueba fue de $14 \pm 4,4^{\circ} \mathrm{C}$ y la humedad relativa fue del $57 \pm 16,1 \%$. En la figura 1 se muestra el perfil del recorrido y el desnivel de la prueba.

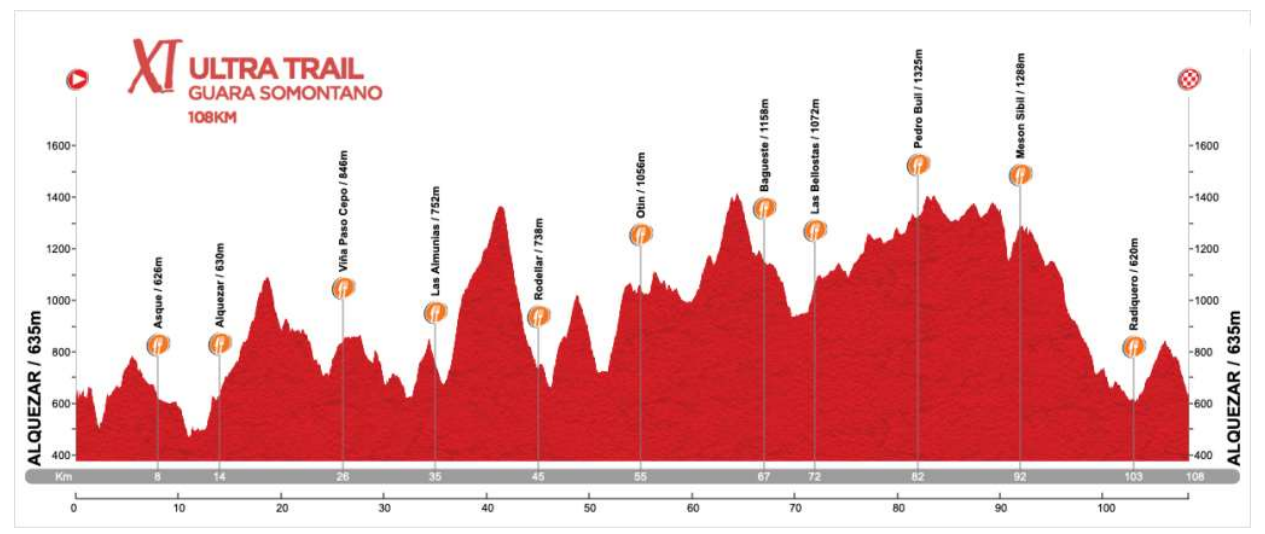

Figura 1. Perfil de la UTGS (30).

\subsection{Muestras de sangre}

Las muestras sanguíneas basales de los sujetos fueron tomadas antes de la realización de la prueba. Las extracciones fueron realizadas por un enfermero insertando una aguja en la vena antecubital. Se utilizaron tubos Vacutainer para extracción de sangre por vacío. Se mantuvieron en condiciones estériles y fueron centrifugadas para, posteriormente, ser transportadas al laboratorio médico para su respectiva conservación (en tubos estériles con heparina de un solo uso) y análisis. El análisis de dichas muestras se realizó mediante análisis químicos automatizados.

Se volvieron a tomar muestras de sangre inmediatamente al finalizar la prueba, para obtener los valores post-carrera utilizando el mismo procedimiento anteriormente comentado. Las muestras se procesaron una hora después de la recogida.

Los parámetros del perfil bioquímico sanguíneo que fueron analizados fueron: $\mathrm{Na}, \mathrm{CPK}$, bilirrubina, ALT, Cr y leucocitos. 


\subsection{Evaluación de la fuerza y composición corporal}

Una semana antes del día de la prueba a los corredores se les realizó una evaluación de la composición corporal y física en el Centro de Medicina del Deporte del Gobierno de Aragón.

Durante esta evaluación, se calculó el peso y la talla de los sujetos, obteniendo el índice de masa corporal (IMC). Posteriormente se calculó la densidad corporal, midiendo los pliegues cutáneos (bicipital, tricipital, subescapular, suprailíaco, abdominal, muslo anterior y pierna medial) con un plicómetro Holtain $®$ (Reino Unido) con una precisión de $0,2 \mathrm{~mm}$. La ecuación que se ha usado para obtener los resultados de porcentaje de masa grasa es la de Yuhasz $(31,32)$.

Tras la evaluación antropométrica se realizó una valoración de la fuerza de las extremidades inferiores mediante test de salto. Se efectuaron tres tipos diferentes de saltos $(33,34)$ : salto desde $90^{\circ}$ o squat jump (SJ), salto con contra movimiento (CMJ) y salto con ayuda de brazos o Abalakov (ABK).

Los saltos se realizaron sobre una plataforma de fuerza Chronojump Bosco-System (España), midiendo el tiempo de vuelo en milisegundos (msg), para poder obtener la altura del salto en centímetros $(\mathrm{cm})$ y la potencia desarrollada en watios $(\mathrm{W})$.

\subsection{Análisis estadístico}

Para realizar el análisis estadístico se utilizó el programa informático de estadística IBM SPSS versión 25 (Estados unidos). Se realizaron análisis descriptivos para conocer las características de todas las variables de la muestra y se realizaron test de normalidad (test de kolmogorov-Smirnov y test de Shapiro-Wilk) para conocer el tipo de distribución que seguían. Posteriormente, para comparar los valores pre carrera con los valores post carrera, y puesto que las variables no seguían una distribución normal, se utilizaron pruebas no paramétricas para muestras relacionadas. Después, se realizaron análisis de varianzas de un factor (ANOVA). Los cofactores que se utilizaron fueron: los años de experiencia en este tipo de carreras, las horas de entrenamiento semanales promedio, el desnivel acumulado de la temporada, cuantos días se realiza trabajo de fuerza, de core y estiramientos, la hidratación en carrera y la pérdida de peso durante la prueba. Las variables dependientes que se utilizaron fueron los valores post carrera de las variables sanguíneas y de saltos y la diferencia entre los valores pre carrera y post carrera de las mismas variables. 


\section{Resultados}

Los valores sanguíneos medios de los nueve sujetos antes y después de la prueba se presentan en la tabla 2. Todas las variables analizadas en sangre sufren un incremento en el transcurso de la carrera, siendo estadísticamente significativa en todas $(p \leq 0,05)$, excepto en el $\mathrm{Na}(p=, 527)$. En el caso de la proteína ALT, los valores promedio de los corredores eran de $20,33 \pm 4,97 \mathrm{UI} / \mathrm{L}$ y tras la prueba de $29,11 \pm 6,47 \mathrm{UI} / \mathrm{L}(p=, 012)$. En el caso de la creatinina, los valores ascienden de $0,81 \pm 0,15 \mathrm{mg} / \mathrm{dL}$ a $1,29 \pm 0,38 \mathrm{mg} / \mathrm{dL}$ $(p=, 008)$. En el de la bilirrubina el aumento producido es de $0,66 \pm 0,24 \mathrm{mg} / \mathrm{dL}$ a $1,14 \pm 0,28 \mathrm{mg} / \mathrm{dL}(p=, 030)$. Los leucocitos ascienden de $5,31 \pm 1,43 \times 10^{9} / \mathrm{L}$ a $13,07 \pm 3,25$ x109/L $(p=, 008)$. Respecto a la CPK los valores ascienden de 155,89 $\pm 65,28 \mathrm{UI} / \mathrm{L}$ a $2492 \pm 844,64 \mathrm{UI} / \mathrm{L}(p=, 008)$. La única variable que no reporta una elevación significativa es el sodio, que asciende de $140 \pm 2,45$ a $141,25 \pm 2,31(p=, 527)$.

Tabla 2: Valores sanguíneos medios pre y post prueba* $(p \leq 0,05)$.

\begin{tabular}{|c|l|l|l|}
\cline { 2 - 4 } \multicolumn{1}{c|}{} & \multicolumn{1}{c|}{ Pre } & \multicolumn{1}{c|}{ Post } & Significación \\
\hline $\mathrm{Na}(\mathrm{mmol} / \mathrm{L})$ & $140 \pm 2,45$ & $141,25 \pm 2,31$ & $p=, 527$ \\
\hline $\mathrm{ALT}(\mathrm{Ul} / \mathrm{L})$ & $20,33 \pm 4,97$ & $29,11 \pm 6,47$ & $p=, 012^{*}$ \\
\hline $\mathrm{CPK}(\mathrm{Ul} / \mathrm{L})$ & $155,89 \pm 65,28$ & $2492 \pm 844,64$ & $p=, 008^{*}$ \\
\hline Creatinina $(\mathrm{mg} / \mathrm{dL})$ & $0,81 \pm 0,15$ & $1,29 \pm 0,38$ & $p=, 008^{*}$ \\
\hline Bilirrubina $(\mathrm{mg} / \mathrm{dL})$ & $0,66 \pm 0,24$ & $1,14 \pm 0,28$ & $p=, 030^{*}$ \\
\hline Leucocitos $\left(\mathrm{x} 10^{9} / \mathrm{L}\right)$ & $5,31 \pm 1,43$ & $13,07 \pm 3,25$ & $p=, 008^{*}$ \\
\hline
\end{tabular}

En relación a los valores obtenidos en las pruebas de salto (Tabla 3), se aprecia que sus resultados disminuyen significativamente al término de la prueba. En el caso del SJ, el valor pasa de $23,8 \pm 2,46$ (pre carrera) a 15,99 $\pm 3,61$ (post carrera) $(p=, 008)$. En el del CMJ, de $27,84 \pm 3,12$ a $18,84 \pm 4,34(p=, 008)$ y en el del $A B K$, de $32,03 \pm 5,50$ a $24,12 \pm 4,82(p=, 008)$.

Tabla 3: valores de salto pre y post prueba * $(p \leq 0,05)$.

\begin{tabular}{|c|c|c|l|}
\cline { 2 - 4 } \multicolumn{1}{c|}{} & Pre & Post & Significación \\
\hline SJ $(m)$ & $23,8 \pm 2,46$ & $15,99 \pm 3,61$ & $p=, 008^{*}$ \\
\hline CMJ $(m)$ & $27,84 \pm 3,12$ & $18,84 \pm 4,34$ & $p=, 008^{*}$ \\
\hline ABK $(m)$ & $32,03 \pm 5,50$ & $24,12 \pm 4,82$ & $p=, 008^{*}$ \\
\hline
\end{tabular}




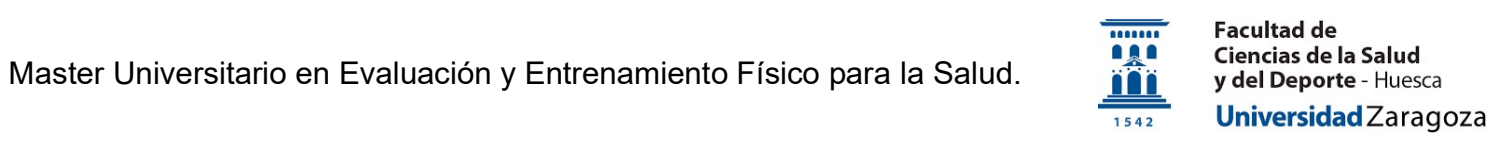

En el test ANOVA, utilizando como cofactor la variable hidratación durante la prueba, se obtienen resultados significativos en la variable salto con contra movimiento post carrera $(p=, 019)$, y, utilizando como cofactor la variable horas de entrenamiento semanales promedio, se han hallado resultados significativos en la variable ALT post carrera $(p=$ ,024).

Realizando el mismo test encontramos que, utilizando el factor horas de entrenamiento semanales promedio, la variable incremento de bilirrubina durante la carrera es estadísticamente significativa $(p=, 022<0,05)$ (figura 2).

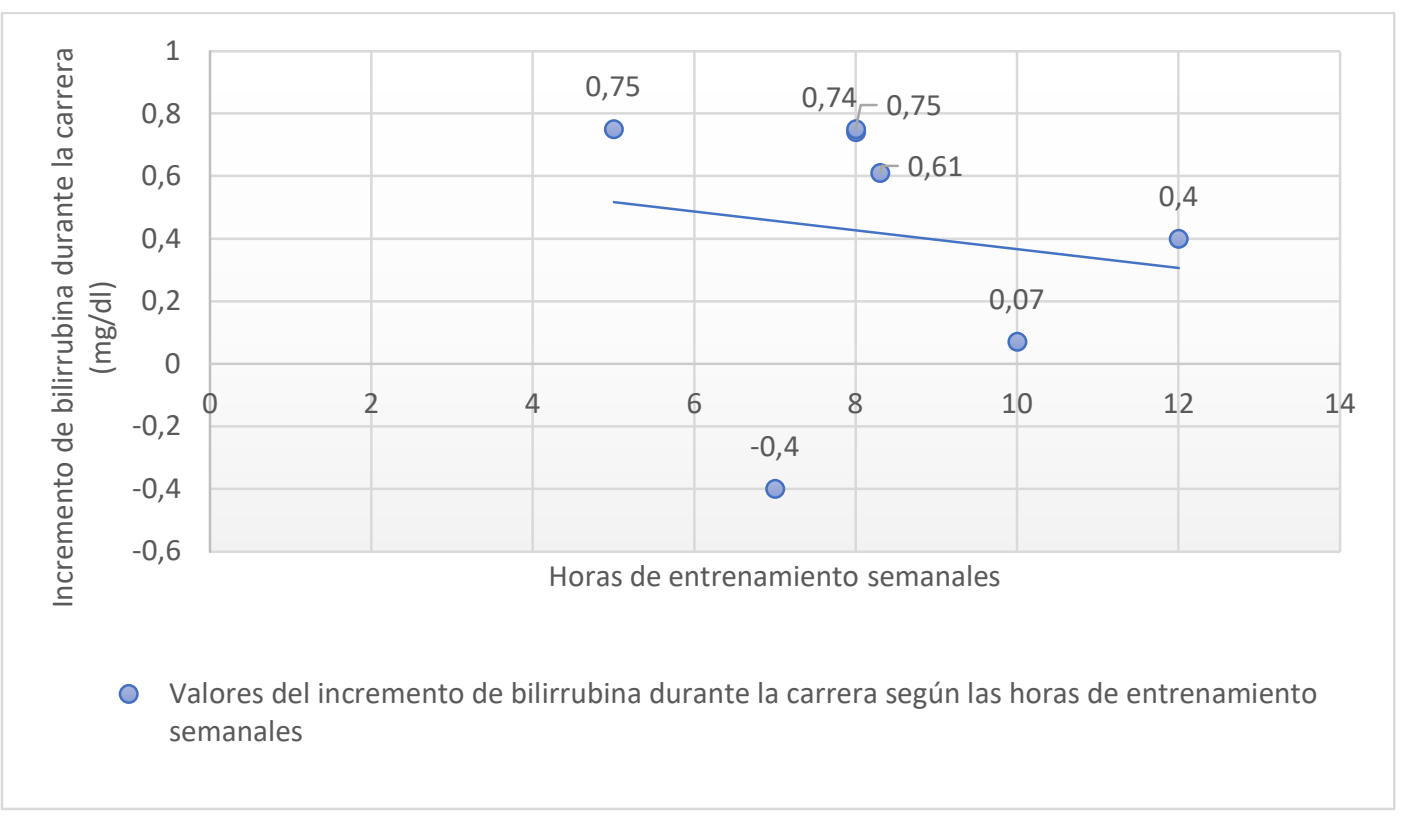

Figura 2. Relación entre el incremento de bilirrubina durante la carrera y horas de entrenamiento semanal 


\section{Discusión}

El objetivo del presente estudio fue analizar el impacto agudo producido en el organismo durante una carrera de montaña en corredores aficionados, analizando diferentes marcadores bioquímicos sanguíneos ( $\mathrm{Na}, \mathrm{CPK}$, bilirrubina, ALT, creatinina y leucocitos) y valorando la posible pérdida de fuerza producida sobre las extremidades inferiores.

La hiponatremia asociada al ejercicio suele estar presente en pruebas de larga distancia (9). Sin embargo, en la muestra analizada los valores medios de $\mathrm{Na}$ aumentan ligeramente. Esta circunstancia puede ser debida a que el día que se realizó la prueba, la temperatura media fue de $14^{\circ} \mathrm{C}$ y la humedad relativa promedio fue del $57 \%$, lo que pudo provocar que los corredores sudaran menos y perdieran menos Na por sudor, de manera similar a lo descrito por Beat Knechtle et al. (35), en donde se indica que en pruebas con temperaturas medias-bajas los casos de hiponatremia debida al ejercicio son escasos.

En el caso de la CPK, los resultados obtenidos se corresponden con los resultados de la mayoría de los estudios (8,15-18), mostrando un daño muscular agudo. El incremento de CPK medio es de 2336,11 UI/L, lo que significa que los corredores podrían padecer rabdomiólisis inducida por el ejercicio ya que el valor medio se sitúa dentro del rango de los valores de referencia para este proceso agudo (18).

Con respecto a la bilirrubina, los valores post carrera son significativamente más altos que los valores basales, tal y como se indica en otros estudios realizados en pruebas de ultramaratón de 24 horas $(10,17,28)$. Este aumento de la bilirrubina sérica es resultado principalmente de la hemólisis que se produce por los continuos impactos que reciben los pies al correr $(10,17)$. Al destruirse los hematíes, la bilirrubina es liberada al torrente sanguíneo y su concentración aumenta.

En referencia a la ALT, no está claro si su incremento podría significar daño hepático. La enzima ALT se encuentra tanto en las células musculares como en las células hepáticas, por lo que su aumento también podría deberse al daño muscular y no al daño hepático $(28,36)$. Aun así, es una de las enzimas que se utiliza como indicador de daño hepático en corredores (36). Los resultados obtenidos coinciden con los resultados encontrados en la literatura $(8,10,17,28,36)$. En este sentido, estudio como el realizado por Skenderi et al. (8), durante la Spartathlon race de $246 \mathrm{~km}$, se evidenciaron incremento sen la ALT, aunque en mayor grado respecto a los resultados hallados en nuestro estudio. Esta diferencia podría deberse a que la distancia recorrida en este estudio es más del doble que en la UTGS. En cambio, en el estudio de Kim et al. (36), sobre una carrera de $200 \mathrm{~km}$, el incremento a los $100 \mathrm{~km}$ se situó en valores similares 
a los hallados en este estudio. En este contexto, aunque existe un incremento de la ALT, no se puede confirmar que se haya producido daño hepático al no haberse incorporado un mayor número de marcadores de daño hepático.

La elevación de los niveles de creatinina en sangre es común entre atletas de ultra maratón (37). Los resultados de los valores de creatinina obtenidos coinciden con los obtenidos en otros estudios (5,37-39), pudiéndose deducir que existe una disminución de la función renal reversible. La causa de esta disfunción podría ser una disminución de la perfusión sanguínea debida a la redistribución del flujo sanguíneo hacia los músculos, o una vasoconstricción arteriolar renal como resultado de los productos de degradación muscular y la rabdomiólisis (39). Como se ha comentado anteriormente, la muestra analizada no ha sufrido una deshidratación elevada, por lo que la disminución de la perfusión por hipovolemia podría ser descartada.

La leucocitosis debida al ejercicio se trata de un fenómeno ampliamente descrito en la literatura científica (12). El aumento de leucocitos que se ha encontrado en los corredores de esta investigación se encuentra en consonancia con la obtenida por Banfi et al. (7), Shin et al., (12) y Nieman et al. (40), con longitudes máximas que varían desde los $48 \mathrm{~km}(\mathrm{n}=82)$, a los $308 \mathrm{~km}$. Estos incrementos no se deben a situaciones infecciosas, sino que se deben a causas multifactoriales. Los principales responsables de provocar una leucocitosis son la inflamación y el daño muscular. El flujo sanguíneo aumentado moviliza leucocitos de las paredes del endotelio y de los principales órganos, que unida al aumento del flujo linfático y al incremento de la actividad simpática que provoca la secreción de catecolaminas, resulta una liberación de grandes cantidades de leucocitos al torrente (21).

El análisis de la fuerza y el daño muscular del tren inferior en una ultramaratón de montaña ha sido objeto de estudio (5), obteniéndose disminución de la capacidad de salto al término de la prueba en las pruebas de salto SJ y CMJ. Esta disminución de la capacidad de salto coincide con lo hallado en esta investigación, aunque es menor que la obtenida en esta investigación. Estas diferencias podrían encontrarse relacionadas con la distancia de la prueba, siendo bastante menor la descrita a la analizada en este estudio, la UTGS, produciéndose probablemente un menor daño muscular.

Estudios como el realizado por del Coso et al. (41), muestran que existe una pérdida de fuerza, medida mediante la altura de salto, tras realizar una maratón que se traduce en una disminución del 20\%. En este sentido, Suzuki et al. (42) realizaron pruebas de SJ y CMJ para valorar el daño muscular en triatletas, obteniéndose resultados muy similares a los obtenidos en esta investigación. Sin embargo, estos datos deben ser tomados con 


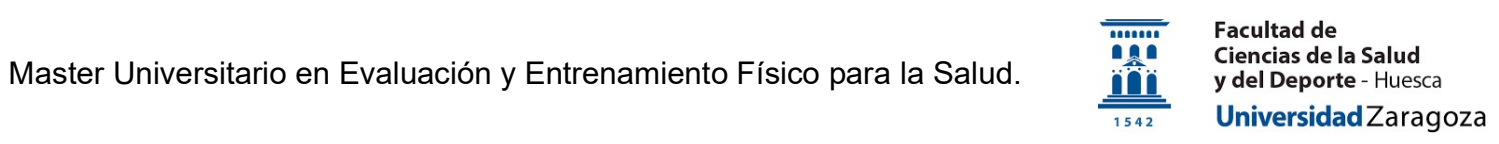

cierta cautela ya que las distancias y el tipo de esfuerzo no es comparable. No obstante, el impacto muscular y la fatiga acumulada en todos los casos ha mermado significativamente la capacidad de salto de los deportistas, afectando probablemente a su integridad musculo tendinosa.

Cuando relacionamos los valores post carrera de las variables con los cofactores anteriormente expuestos en los resultados, parece existir una tendencia positiva entre la hidratación durante la prueba y el salto con contra movimiento, significando que, cuanto más líquidos ingieren los atletas, menor es la perdida de salto que se produce al término de la prueba. Esta relación coincide con lo descrito en la literatura $(43,44)$, puesto que una situación de hipohidratación puede disminuir la capacidad para generar fuerza y potencia, afectando a la función neuromuscular, al equilibrio acido-base corporal y a la actividad cardiovascular y metabólica (44).

También se ha podido observar una tendencia positiva entre las horas de entrenamiento semanales y la ALT, dando como resultado que cuantas más horas de entrenamiento realizaban los sujetos, mayores son los valores de ALT post carrera. Esta correlación no se corresponde a lo que aparece en la literatura científica, puesto que la ALT es un marcador de daño muscular, y cuanto más entrenado está un corredor, mayor es la capacidad de sus fibras musculares para soportar la carga de trabajo exigida (22-25). Por lo tanto, cuanto más entrenado este el corredor, más adaptado estará y menos daño muscular sufrirá.

En este contexto, al analizar los parámetros sanguíneos y de fuerza de tren inferior, teniendo en cuenta los valores pre y post carrera y relacionándolos con los cofactores utilizados, el único resultado significativo que hemos obtenido es la relación entre las horas de entrenamiento y la bilirrubina. Parece existir una relación inversamente proporcional entre las horas de entrenamiento semanales y el incremento de bilirrubina, es decir, a mayor número de horas de entrenamiento, menor es el aumento que se produce de bilirrubina en sangre debido a la carrera. Esto podría ser debido a que cuantas más horas de entrenamiento se realiza, la adaptación del organismo a ese esfuerzo es mayor (22-25), y éste es capaz de adaptarse a una situación de repetidos impactos disminuyendo el porcentaje de sangre hemolizada. 


\section{Conclusiones}

A la vista de los resultados obtenidos y según nuestros objetivos, podemos concluir que:

- La CPK, la ALT, la bilirrubina, la creatinina y los leucocitos, aumentan significativamente en sangre después de una ultramaratón de montaña, mientras que el $\mathrm{Na}$, no muestra una variación significativa.

- El incremento de la CPK en el torrente sanguíneo nos indica la existencia de daño muscular y de rabdomiólisis inducida por ejercicio. El aumento de ALT también nos indica la existencia de daño muscular, pero, además, la posible existencia de daño hepático.

- El incremento de bilirrubina nos indica una hemólisis aumentada, provocada por los continuos impactos de las extremidades inferiores contra el suelo durante la carrera.

- El aumento de creatinina nos muestra una posible disfunción renal producida por la redistribución sanguínea hacia los músculos y a la acumulación de sustancias de desecho en la sangre provocadas por la ruptura de células musculares.

- La leucocitosis provocada por el ejercicio es producida por el aumento del flujo sanguíneo y por el aumento de citoquinas producido por el daño muscular.

- No se ha producido hiponatremia inducida por el ejercicio debido a la temperatura media de la prueba y a la correcta hidratación de los corredores.

- Existe pérdida de capacidad de salto en los corredores tras la prueba debido a la afectación musculo tendinosa y neuromuscular de las extremidades inferiores.

- Se ha encontrado una relación positiva entre la capacidad de salto en la prueba CMJ post carrera y la hidratación durante la misma y entre las horas de entrenamiento semanales promedio y la ALT post carrera.

- También, se ha encontrado una relación inversamente proporcional entre las horas de entrenamiento semanales promedio y el incremento de bilirrubina durante la carrera. 


\section{Limitaciones y prospectivas}

En este trabajo se puede apreciar que durante una prueba de ultramaratón de montaña, los corredores pueden llegar a sufrir disfunción aguda renal y hepática, daño muscular agudo, hemólisis y disminución de la capacidad contráctil; además, por su carácter novedoso, invita a seguir esta línea de investigación para conocer si los daños que se producen en el organismo pueden llegar a ser crónicos, o para realizar comparaciones entre el impacto que tienen estas pruebas en deportistas aficionados y en corredores de élite o entre atletas femeninas y masculinos.

Este trabajo presenta algunas limitaciones. La primera de ellas, que posiblemente condicione a las demás, es el reducido número de personas que conforman la muestra. Si bien es cierto que es similar a otros estudios consultados, posiblemente con un mayor número de corredores en nuestro análisis, podríamos haber obtenido más resultados significativos, como muestran estudios realizados en pruebas con características parecidas a la que hemos analizado.

Los resultados que se han obtenido teniendo en cuenta únicamente los valores post carrera son menos representativos que los resultados que tienen en cuenta los valores pre y post carrera, puesto que cuando se tienen en cuenta ambos valores los resultados son no significativos. Desconocemos si esto podría deberse al pequeño tamaño muestral o a otras razones, por lo que debería ser uno de los objetos de estudio en trabajos posteriores.

Por otra parte, podemos señalar como una limitación añadida no haber medido otro marcador de daño hepático para poder compararlo con ALT y valorar si realmente se produce daño hepático en los corredores o el aumento de ALT en sangre proviene de la rabdomiólisis producida por el daño muscular. 
Master Universitario en Evaluación y Entrenamiento Físico para la Salud.

\section{Referencias}

1. Cejka N, Rüst CA, Lepers R, Onywera V, Rosemann T, Knechtle B. Participation and performance trends in 100-km ultra-marathons worldwide. J Sports Sci. 2014;32(4):354-66.

2. Scheer V. Participation trends of ultra endurance events. Sports Med Arthrosc. 2019;27(1):3-7.

3. Javier Ullé. Los orígenes del trail running. 2019.

4. Escalada FE de D de M y. FEDME. 2013.

5. Ramos-campo DJ, Ávila-gandía V, Alacid F, Alcaraz PE, López-román FJ RJ. Muscle damage, physiological changes and energy balance in ultra-endurance mountain event athletes. J Chem Inf Model. 2016;53(9):1689-99.

6. Kreider RB. Physiological considerations of ultraendurance performance. $1991 ; 3-27$.

7. Banfi G, Roi GS, Dolci A, Susta D. Behaviour of haematological parameters in athletes performing marathons and ultramarathons in altitude ('skyrunners'). Clin Lab Haematol. 2004;26(6):373-7.

8. Skenderi KP, Kavouras SA, Anastasiou CA, Yiannakouris N, Matalas AL. Exertional rhabdomyolysis during a 246-km continuous running race. Med Sci Sports Exerc. 2006;38(6):1054-7.

9. Chlíbková D, Rosemann T, Posch L, Matoušek R, Knechtle B. Pre- and postrace hydration status in hyponatremic and non-hyponatremic ultra-endurance athletes. Chin J Physiol. 2016;59(3):173-83.

10. Wu HJ, Chen KT, Shee BW, Chang HC, Huang YJ, Yang R Sen. Effects of $24 \mathrm{~h}$ ultra-marathon on biochemical and hematological parameters. World $\mathrm{J}$ Gastroenterol. 2004;10(18):2711-4.

11. Rojas-Valverde D, Sánchez-Ureña B, Pino-Ortega J, Gómez-Carmona C, Gutiérrez-Vargas R, Timón R, et al. External workload indicators of muscle and kidney mechanical injury in endurance trail running. Int $\mathrm{J}$ Environ Res Public Health. 2019;16(20):1-13.

12. Shin YO, Lee JB. Leukocyte chemotactic cytokine and leukocyte subset responses during ultra-marathon running. Cytokine. 2013;61(2):364-9. 
13. Hew-Butler T, Jordaan E, Stuempfle KJ, Speedy DB, Siegel AJ, Noakes TD, et al. Osmotic and nonosmotic regulation of arginine vasopressin during prolonged endurance exercise. J Clin Endocrinol Metab. 2008;93(6):2072-8.

14. Montain SJ, Sawka MN, Wenger CB. Hyponatremia associated with exercise: Risk factors and pathogenesis. Exerc Sport Sci Rev. 2001;29(3):113-7.

15. Brancaccio P, Lippi G, Maffulli N. Biochemical markers of muscular damage. Clin Chem Lab Med. 2010;48(6):757-67.

16. Magrini D, Khodaee M, San-Millán I, Hew-Butler T, Provance AJ. Serum creatine kinase elevations in ultramarathon runners at high altitude. Phys Sportsmed. 2017;45(2):129-33.

17. Shin KA, Park KD, Ahn J, Park Y, Kim YJ. Comparison of Changes in Biochemical Markers for Skeletal Muscles, Hepatic Metabolism, and Renal Function after Three Types of Long-distance Running. Med (United States). 2016;95(20):1-6.

18. Rojas-valverde D, Sánchez-ureña B, Crowe J, Timón R, Olcina GJ. Exertional rhabdomiolysis and acute kidney injury in endurance sports: A systematic review. Eur J Sport Sci. 2020;0(0):1-28.

19. Artrong RB. Muscle Damage and Endurance Events. Sport Med. 1986;3(5):37081.

20. Linda S. Costanzo. Fisiología. Elsevier Inc; 2014. 254-260 p.

21. Simpson RJ, Kunz H, Agha N, Graff R. Exercise and the Regulation of Immune Functions. 1st ed. Vol. 135, Progress in Molecular Biology and Translational Science. Elsevier Inc.; 2015. 355-380 p.

22. Denadai BS, de Aguiar RA, de Lima LCR, Greco CC, Caputo F. Explosive Training and Heavy Weight Training are Effective for Improving Running Economy in Endurance Athletes: A Systematic Review and Meta-Analysis. Sport Med. 2017;47(3):545-54.

23. Giovanelli N, Taboga P, Rejc E, Lazzer S. Effects of strength, explosive and plyometric training on energy cost of running in ultra-endurance athletes. Eur $\mathrm{J}$ Sport Sci. 2017;17(7):805-13.

24. Rønnestad BR, Mujika I. Optimizing strength training for running and cycling endurance performance: A review. Scand J Med Sci Sport. 2014;24(4):603-12. 
25. Berryman N, Mujika I, Arvisais D, Roubeix M, Binet C, Bosquet L. Strength training for middle- and long-distance performance: A meta-analysis. Int J Sports Physiol Perform. 2018;13(1):57-63.

26. Knechtle B, Nikolaidis PT. Physiology and pathophysiology in ultra-marathon running. Front Physiol. 2018;9(JUN).

27. Scheer BV, Murray A. Al andalus ultra trail: An observation of medical interventions during a 219-km, 5-day ultramarathon stage race. Clin J Sport Med. 2011;21(5):444-6.

28. Tirabassi JN, Olewinski L, Khodaee M. Variation of Traditional Biomarkers of Liver Injury After an Ultramarathon at Altitude. Sports Health. 2018;10(4):361-5.

29. Fornasiero A, Savoldelli A, Fruet D, Boccia G, Pellegrini B, Schena F. Physiological intensity profile, exercise load and performance predictors of a 65km mountain ultra-marathon. J Sports Sci. 2018;36(11):1287-95.

30. UTGS.es. Perfil y desnivel de la UTGS. 2020.

31. Yuhasz Ms. Physical fitness manual. Ontario: University of Western Ontario.; 1974.

32. G. Gris. Componentes del somatotipo y ecuaciones antropométricas. Apunt Educ Física y Deport. 2016;12.

33. Garrido R, González M, Expósito I, Sirvent J, García M. Valores del Test de Bosco en Función del Deporte. PubliCE. 2012;(1998):1-20.

34. Valero H, Suárez J. Análisis de la evaluación de potencia en tren inferior: una revisión sistemática. Act Física y Deport. 2017;3(2):13-24.

35. Knechtle B, Chlíbková D, Papadopoulou S, Mantzorou M, Rosemann T, Nikolaidis PT. Exercise-associated hyponatremia in endurance and ultraendurance performance-aspects of sex, race location, ambient temperature, sports discipline, and length of performance: a narrative review. Med. 2019;55(9):1-23.

36. Kim HJ, Lee YH, Kim CK. Biomarkers of muscle and cartilage damage and inflammation during a 200 km run. Eur J Appl Physiol. 2007;99(4):443-7.

37. Khodaee M, Spittler J, Vanbaak K, Changstrom BG, Hill JC. Effects of Running an Ultramarathon on Cardiac, Hematologic, and Metabolic Biomarkers. Int J Sports Med. 2015;36(11):867-71. 
Master Universitario en Evaluación y Entrenamiento Físico para la Salud.

38. Wołyniec W, Ratkowski W, Kasprowicz K, Jastrzębski Z, Małgorzewicz S, Witek $\mathrm{K}$, et al. Glomerular filtration rate is unchanged by ultramarathon. J Strength Cond Res. 2018;32(11):3207-15.

39. Hoffman MD, Weiss RH. Does acute kidney injury from an ultramarathon increase the risk for greater subsequent injury? Clin J Sport Med. 2016;26(5):417-22.

40. Nieman DC, Dumke CL, Henson DA, McAnulty SR, Gross SJ, Lind RH. Muscle damage is linked to cytokine changes following a 160-km race. Brain Behav Immun. 2005;19(5):398-403.

41. Del Coso J, Salinero JJ, Abián-Vicen J, González-Millán C, Garde S, Vega P, et al. Influence of body mass loss and myoglobinuria on the development of muscle fatigue after a marathon in a warm environment. Appl Physiol Nutr Metab. 2013;38(3):286-91.

42. Suzuki K, Peake J, Nosaka K, Okutsu M, Abbiss CR, Surriano R, et al. Changes in markers of muscle damage, inflammation and HSP70 after an Ironman triathlon race. Eur J Appl Physiol. 2006;98(6):525-34.

43. Pallarés JG, Martínez-Abellán A, López-Gullón JM, Morán-Navarro R, De la Cruz-Sánchez E, Mora-Rodríguez R. Muscle contraction velocity, strength and power output changes following different degrees of hypohydration in competitive olympic combat sports. J Int Soc Sports Nutr [Internet]. 2016;13(1):1-9.

44. Judelson DA, Maresh CM, Anderson JM, Armstrong LE, Casa DJ, Kraemer WJ, et al. Hydration and muscular performance: Does fluid balance affect strength, power and high-intensity endurance? Sport Med. 2007;37(10):907-21. 